\title{
Validação da Escala de Estresse no Trabalho
}

\author{
Tatiane Paschoal \\ Álvaro Tamayo \\ Universidade de Brasília
}

\begin{abstract}
Resumo
Este trabalho teve como objetivo construir e validar um instrumento de estresse ocupacional geral, de fácil aplicação e que pudesse ser utilizado em diversos ambientes de trabalho e para ocupações variadas. A Escala de Estresse no Trabalho (EET), inicialmente composta por 31 itens, foi aplicada a 437 trabalhadores de diferentes organizações, públicas e privadas, sendo 249 homens e 188 mulheres. A análise fatorial revelou a existência de um único fator que, após eliminação de itens com carga fatorial abaixo de 0,45 , ficou composto por 23 itens e obteve um coeficiente alfa de Cronbach equivalente a 0,91. Uma versão reduzida da escala, com 13 itens e alfa de 0,85 foi proposta. Com base nos parâmetros psicométricos satisfatórios da EET, concluise que esta é uma alternativa para investigações empíricas e trabalhos aplicados em organizações, podendo orientar medidas que visem à qualidade de vida dos trabalhadores.
\end{abstract}

Palavras-chave: estresse ocupacional; estressores organizacionais; psicometria

\begin{abstract}
Validation of the work stress scale. The objective of this study was to develop and to validate a general instrument to evaluate occupational stress, easily administered, used in different work environments and for a variety of occupations. The Work Stress Scale (WSS) initially composed of 31 items was applied to 437 workers, 249 men and 188 women, of public and private organizations. The factor analysis detected only one factor. After the elimination of the items with a factor loading below .45, the final version of the scale was composed of 23 items and had a Cronbach's coefficient of .91. A reduced version of the scale with 13 items and a Cronbach's coefficient of .85 has been proposed. Based on the satisfactory psychometric parameters of the scale, it is concluded that the WSS is useful as an alternative in empirical investigations and organizational diagnostics. The results obtained can be useful indicators for organizational decisions in relation to the life quality of the workers.
\end{abstract}

Key words: occupational stress; organizational stressors; psychometrics

$\mathrm{O}$ interesse pelo estudo do estresse no trabalho tem sido crescente na literatura científica, particularmente nos últimos anos. Uma razão para o aumento de pesquisas sobre este tema deve-se ao impacto negativo do estresse ocupacional na saúde e no bem-estar dos empregados e, conseqüentemente, no funcionamento e na efetividade das organizações. Na economia, o impacto negativo dessa variável tem sido estimado com base na suposição e nos achados de que trabalhadores estressados diminuem seu desempenho e aumentam os custos das organizações com problemas de saúde, com o aumento do absenteísmo, da rotatividade e do número de acidentes no local de trabalho (Jex, 1998). Numa época de intensa competitividade, tanto entre organizações quanto entre profissionais, diversas pesquisas têm sido realizadas sobre a natureza e os mecanismos do estresse ocupacional e sobre suas conseqüências para a saúde e desempenho do empregado (Cavanaugh, Boswell, Roehling, \& Boudreau , 2000; Cohen, Kessler, \& Gordon, 1995;
Evans \& Steptoe, 2001; Fried, Ben-David, Tiegs, Avital, \& Yeverechyahu, 1998; Lim, Ong, \& Phoon, 1987; Mackie, Holahan, \& Gottlieb, 2001; Pollard, 2001; Wang \& Patten, 2001).

Em meio à multiplicidade de pesquisas, nota-se que o termo estresse ocupacional tem sido utilizado de modo pouco consistente, havendo desentendimentos sobre seu significado e formas de medição. Segundo Jex (1998), as definições de estresse ocupacional dividem-se de acordo com três aspectos: (1) estímulos estressores: estresse ocupacional refere-se aos estímulos do ambiente de trabalho que exigem respostas adaptativas por parte do empregado e que excedem a sua habilidade de enfrentamento (coping); estes estímulos são comumente chamados de estressores organizacionais; (2) respostas aos eventos estressores: estresse ocupacional refere-se às respostas (psicológicas, fisiológicas e comportamentais) que os indivíduos emitem quando expostos a fatores do trabalho que excedem sua ha- 
bilidade de enfrentamento; (3) estímulos estressores-respostas: estresse ocupacional refere-se ao processo geral em que demandas do trabalho têm impacto nos empregados.

De acordo com a definição priorizada, portanto, os estudos podem se basear nos estressores organizacionais, nas respostas do indivíduo a esses estressores ou nas diversas variáveis presentes no processo estressor-resposta.

A conceituação do estresse ocupacional a partir do enfoque nos estressores organizacionais permite diferenciar dois tipos de estudo: os de estresse ocupacional e os de estresse em geral. Os primeiros enfocam estressores relacionados ao ambiente de trabalho e os últimos, estressores gerais na vida do indivíduo. A abordagem que enfoca os estressores organizacionais têm contribuído para a identificação de demandas organizacionais potencialmente geradoras de estresse. Apesar das contribuições, esta abordagem tende a considerar o caráter objetivo dos estressores e tem sido alvo de inúmeras críticas. Segundo Lazarus (1995) e Lazarus e Folkman (1984), a simples presença de eventos que podem se constituir como estressores em determinado contexto, no qual o indivíduo esteja inserido, não caracteriza um fenômeno de estresse. Para que isto ocorra, é necessário que o indivíduo perceba e avalie os eventos como estressores, o que quer dizer que fatores cognitivos têm um papel central no processo que ocorre entre os estímulos potencialmente estressores e as respostas do indivíduo a eles. A existência de um evento potencialmente estressor na organização não quer dizer que ele será percebido desta forma pelo indivíduo. Por exemplo, um trabalhador que relata a existência de excesso de trabalho pode não percebê-la como prejudicial, mas sim, como positiva e estimulante. Características situacionais e pessoais podem interferir no julgamento do indivíduo. Assim, definir estresse ocupacional como estressores organizacionais deixa uma lacuna relativa à avaliação do indivíduo sobre os eventos do trabalho.

Quanto à definição do estresse ocupacional a partir das respostas aos eventos estressores, pode-se apontar sua contribuição para a identificação e compreensão de conseqüências do estresse. A principal crítica a esta abordagem refere-se à dificuldade em estabelecer se determinados comportamentos, estados afetivos e problemas de saúde são conseqüências de estressores organizacionais ou de outros contextos e eventos da vida do indivíduo (Jones \& Kinman, 2001; Kahn \& Byosiere, 1992).

Ultimamente, os autores têm preferido considerar definições mais abrangentes e que estejam menos suscetíveis às críticas anteriores. Para Beehr (1998), por exemplo, o estresse ocupacional consiste num fenômeno tão complexo que não deveria ser tratado como uma variável, mas como uma área de estudo e prática que se preocupa com diversas variáveis interligadas, tais como estímulos do ambiente de trabalho e respostas não saudáveis de pessoas expostas a eles.

De modo geral, há uma tendência em se considerar o estresse ocupacional como um processo estressores-respostas, já que este consiste no enfoque mais completo e engloba aquele baseado nos estressores e o baseado nas respostas.
Apesar das peculiaridades de cada tipo de definição e de modelos existentes para explicar o estresse ocupacional, temse constatado um consenso entre os estudiosos da área de que as percepções dos indivíduos são mediadoras do impacto do ambiente de trabalho sobre o indivíduo; para algo na organização ser um estressor, ele precisa ser percebido como tal pelo empregado. O estresse ocupacional pode ser definido, portanto, como um processo em que o indivíduo percebe demandas do trabalho como estressores, os quais, ao exceder sua habilidade de enfrentamento, provocam no sujeito reações negativas.

A seguir, apresenta-se uma breve revisão dos principais estressores organizacionais apontados na literatura, respostas dos indivíduos a estes estressores e variáveis que influenciam o estresse ocupacional.

Em relação aos estressores organizacionais, estes podem ser de natureza física (por exemplo, barulho, ventilação e iluminação do local de trabalho) ou psicossocial, sendo que os últimos têm despertado maiores interesses nos psicólogos organizacionais. Entre os estressores psicossociais, destacam-se os estressores baseados nos papéis, os fatores intrínsecos ao trabalho, os aspectos do relacionamento interpessoal no trabalho, a autonomia/controle no trabalho e os fatores relacionados ao desenvolvimento da carreira.

Grande parte dos estudos que enfoca estressores organizacionais tem se apoiado nas teorias de papéis. Dois fatores principais constituem esta categoria de estressores. O primeiro refere-se ao conflito entre papéis, o qual ocorre quando informações advindas de um membro ou contexto do trabalho entra em conflito com as informações de outro membro ou contexto (Jex, 1998). O outro estressor associado aos papéis refere-se à ambigüidade do papel. Neste caso, as informações associadas ao papel que o empregado deve desempenhar são pouco claras e inconsistentes (Jex, 1998).

De acordo com Glowinkowski e Cooper (1987), os estressores podem ser fatores intrínsecos ao trabalho, os quais se referem a aspectos como repetição de tarefas, pressões de tempo e sobrecarga. Dentre eles, a sobrecarga de trabalho tem recebido considerável atenção dos pesquisadores. Este estressor pode ser dividido em dois níveis: quantitativo e qualitativo. A sobrecarga quantitativa diz respeito ao número excessivo de tarefas a serem realizadas; isto é, a quantidade de tarefas encontra-se além da disponibilidade do trabalhador. A sobrecarga qualitativa refere-se à dificuldade do trabalho, ou seja, o indivíduo depara-se com demandas que estão além de suas habilidades ou aptidões (Glowinkowski \& Cooper, 1987; Jex, 1998).

Outra categoria de estressores refere-se ao relacionamento interpessoal no trabalho. A grande maioria das ocupações envolve interações entre pessoas, seja entre colegas de mesmo nível hierárquico, superiores e subordinados, seja entre empregados e clientes. Quando essas interações resultam em conflitos tem-se uma outra fonte de estresse (Glowinkowski \& Cooper, 1987; Jex, 1998).

$\mathrm{Na}$ categoria dos fatores relacionados ao desenvolvimento da carreira, Glowinkowski e Cooper (1987) destacam 
os aspectos relacionados à falta de estabilidade no trabalho, ao medo de obsolescência frente às mudanças tecnológicas e às poucas perspectivas de promoções e crescimento na carreira.

Finalmente, outro tipo de estressor, comumente relatado na literatura, refere-se ao controle/autonomia no trabalho. Segundo Kahn e Byosiere (1992), o controle tem sido tratado em termos da autonomia do trabalhador em relação às decisões e aos métodos de trabalho.

Dependendo de suas percepções, o indivíduo pode reagir aos estressores organizacionais de forma positiva ou negativa. Neste último caso, tem-se o fenômeno de strain, termo que abrange a diversidade de respostas negativas que são emitidas como conseqüência da exposição aos estressores. As categorias de resposta aos estressores podem ser divididas em: psicológicas, fisiológicas e comportamentais.

Fundamentalmente, as reações associadas a estressores são de natureza emocional, sendo que as respostas psicológicas aos estressores organizacionais são comuns na literatura. Cooper e Cartwright (2001) referem-se às emoções destrutivas e ao estresse como se fossem um único construto e afirmam que, no futuro, o estudo do estresse no trabalho poderá ser substituído pelo estudo das emoções no trabalho. Stanley e Burrows (2001) incluem o estresse no elenco das principais emoções do adulto, ainda que afirmem que o estresse consiste num processo e não estritamente numa emoção. Para estes autores, a ativação psicológica ou física causada pela discrepância entre demandas situacionais e mecanismos de enfrentamento leva a uma gama de emoções como tensão, ansiedade, irritabilidade, nervosismo e raiva, entre outras.

Muitas das respostas psicológicas enfocadas nos estudos sobre estresse ocupacional têm consistido na insatisfação no trabalho, na ansiedade e na depressão. Wang e Patten (2001) verificaram que quanto maior o estresse ocupacional, mais sintomas de depressão os indivíduos apresentam. Mackie, Holahan e Gottlieb (2001) observaram a relação entre determinadas práticas gerenciais, percepção de estressores no trabalho e sintomas de depressão por parte dos empregados. Cavanaugh, Boswell, Roehling e Boudreau (2000) verificaram que a percepção de estressores organizacionais que dificultam ou impedem o desempenho de tarefas valorizadas estava negativamente relacionada com a satisfação no trabalho. Tamayo, Paschoal, Rego e Ferreira (2002) investigaram a relação entre o estresse ocupacional e o autoconceito profissional. Os resultados mostraram que o estresse ocupacional se correlacionou negativamente com a percepção de saúde no trabalho, segurança e competência profissional.

Até agora, foram apresentados os principais estressores organizacionais e respostas do indivíduo presentes na literatura. Em quase todos os estudos, encontramse também inúmeras variáveis de natureza pessoal e situacional que podem influenciar o estresse ocupacional, independentemente da definição adotada para este construto.
Quanto às variáveis situacionais, destaca-se o suporte social encontrado pelo trabalhador, o qual pode ser recebido do supervisor, dos colegas de trabalho ou mesmo de pessoas fora do trabalho. Iwata e Suzuki (1997) estudaram a relação do suporte social de supervisores, colegas, de membros da família e de outras pessoas significativas com o estresse ocupacional e observaram que o suporte dos supervisores e dos outros significativos se relacionou negativamente ao estresse. Quanto às variáveis pessoais que influenciam o estresse ocupacional, o estilo de enfrentamento (estilo de coping) do empregado frente aos eventos estressores consiste na principal variável individual, representando ainda, um ponto central de muitos estudos. Outras variáveis, como o padrão de comportamento tipo A, o locus de controle e a auto-estima, aparecem com relativa freqüência entre as variáveis pessoais apontadas como capazes de influenciar o processo de estresse (Caplan \& Jones, 1975; Jamal, 1990; Jex \& Elacqua, 1999; Krause \& Stryker, 1984; Marino \& White, 1985).

Em relação aos instrumentos, o problema da medida ou avaliação do estresse ocupacional tem constituído um dos grandes desafios dos pesquisadores. O paradigma geral utilizado nos instrumentos de avaliação do estresse consiste em apresentar ao indivíduo uma lista de estressores, que devem ser avaliados numa escala de freqüência, e uma lista de reações, que devem também ser avaliadas numa escala, independentemente dos estressores. Numerosas preocupações têm sido manifestadas por diversos autores em relação aos instrumentos de medida existentes e aos modelos utilizados na sua construção. A abordagem teórica de Lazarus (1995), segundo a qual o estresse ocupacional é um processo que implica numa transação entre o empregado e o seu ambiente de trabalho, tem dado origem a críticas bastante relevantes, particularmente no que concerne à suposta objetividade dos estressores.

Mesmo entre a diversidade de definições e modelos, a grande maioria das abordagens concorda com o pressuposto de que as percepções são mediadoras do impacto do ambiente de trabalho (Jex, 1998; Lazarus, 1995). No entanto, a maior parte das escalas de estresse ocupacional aborda os estressores, sem solicitar sua avaliação por parte dos sujeitos, e as reações físicas e psicológicas aos estressores, sem que o próprio sujeito estabeleça as possíveis relações entre as duas medidas.

A principal restrição em relação às medidas de estressores é decorrente das críticas sobre as definições do estresse ocupacional a partir dos estressores organizacionais, ou seja, nem sempre eventos considerados estressores nos instrumentos de medida são avaliados desta forma pelo empregado (Jones \& Kinman, 2001; Kahn \& Byosiere, 1992). A significação dos estressores para os empregados pode estar sendo, freqüentemente, ignorada (Cooper, Dewe, \& O’Driscoll, 2001). Outra limitação deste tipo de medida refere-se à desatualização dos instrumentos, que não levam em consideração as transformações significativas ocorridas recentemente no mundo do trabalho e das organizações. Freqüentemente, são considerados tipos particulares de estressores, sendo 
comuns os de conflito entre papéis, ambigüidade de papéis e sobrecarga de trabalho. Desta forma, a importância de certos estressores pode estar sendo superenfatizada, ao passo que outros estressores potenciais, atualmente relevantes, como o assédio moral, o medo de perder o emprego, perspectivas de privatização e mesmo estressores baseados no desenvolvimento da carreira, na autonomia/controle no trabalho e no relacionamento interpessoal podem estar ausentes nos instrumentos de medida.

Quanto às medidas de reações aos estressores, a principal crítica dirige-se à definição do estresse ocupacional como respostas a estressores; isto é, não se pode afirmar que os sintomas investigados sejam conseqüências de estressores organizacionais. Variáveis como problemas gastrintestinais, disfunções cardíacas, insônia, insatisfação no trabalho, absenteísmo, ansiedade e irritação, por exemplo, têm sido apontadas como conseqüências de estressores organizacionais, mas podem ocorrer devido a fatores diversos (Jones \& Kinman, 2001; Kahn \& Byosiere, 1992).

De modo geral, os questionários para investigar o estresse ocupacional têm sido utilizados por conseguirem captar informações de um grande número de sujeitos e produzirem resultados quantificáveis, que permitem comparações mais objetivas entre indivíduos (Quick, Quick, Nelson, \& Hurrell, 1997).

A revisão da literatura sobre medidas de estresse ocupacional utilizadas no Brasil apontou dois instrumentos que têm guiado os estudos na área: o OSI (Occupational Stress Indicator) e o SWS Survey (Questionário de Estresse, Saúde Mental e Trabalho).

O primeiro foi desenvolvido originalmente por Cooper, Sloan e Williams (1988) e tenta acessar os inúmeros construtos presentes no complexo processo do estresse ocupacional, incluindo medidas de estressores, mediadores e conseqüências físicas e psicológicas. Ao todo, são 167 itens agrupados em 25 fatores. A validação deste instrumento tem sido alvo de inúmeras críticas. Três problemas principais podem ser levantados: a) não foi realizada análise fatorial de todas as escalas, sendo que a divisão de alguns fatores ficou baseada na suposição dos autores, b) em relação ao índice de confiabilidade, algumas escalas obtiveram um coeficiente alfa muito abaixo do aceitável, e c) o tamanho da amostra utilizada, de aproximadamente 200 sujeitos, é inadequado para sua validação fatorial (Lyne, Barret, Williams, \& Coaley, 2000). Estudos recentes sobre as propriedades psicométricas do OSI, em que se realizou análise fatorial das escalas, não confirmaram a suposição inicial dos autores sobre os fatores que compõem o instrumento (Evers, Frese, \& Cooper, 2000; Lyne et al., 2000).

Swan, Moraes e Cooper (1993) realizaram a tradução dos itens do OSI para a língua portuguesa e conduziram um estudo para detectar a validade do instrumento no Brasil. A partir daí, as escalas traduzidas do OSI começaram a ser utilizadas em pesquisas brasileiras. A tentativa de validação do OSI no Brasil, porém, preocupa em relação ao tamanho da amostra utilizada: 84 sujeitos. Considerando o número de itens do instrumento ou mesmo o número de fatores que compõem cada escala, a quantidade de sujeitos para a validação é insuficiente (Pasquali, 1999). Apesar de ser uma medida bastante utilizada em diversos países, no Brasil o OSI ainda carece de dados sobre sua adequação e validade.

O segundo instrumento disponível no Brasil, a versão brasileira do SWS - Questionário de Estresse, Saúde Mental e Trabalho (Guimarães \& McFadden, 1999), é composto por 184 itens fechados, distribuídos em oito escalas: fatores psicossociais de risco, saúde mental, estresse no trabalho, apoio no trabalho, estresse social, apoio social, estresse pessoal e apoio pessoal. O fator estresse no trabalho, apresentado para medir especificamente esse construto, contém 26 itens. Em relação à validação do instrumento no Brasil (Guimarães \& McFadden, 1999), o relato da pesquisa apresenta aspectos da análise de juízes e da análise semântica dos itens (sua aplicabilidade para o contexto brasileiro), mas não menciona a validação da escala. A falta de tais informações dificulta o estabelecimento de conclusões seguras a respeito da versão brasileira do SWS. Além disso, a escala de concordância para respostas é dicotômica, cujas possibilidades de pontuação são sim e não.

Apesar do aumento de estudos sobre estresse ocupacional, especialmente na década de 90, e da existência no mercado de numerosos instrumentos para a sua avaliação, poucos são aqueles que atendem os parâmetros psicométricos e os postulados teóricos. Em relação ao Brasil, a situação é complicada, pois os dois instrumentos em circulação apresentam problemas de validação.

Este estudo teve como objetivo construir e validar um instrumento de estresse ocupacional geral, baseado num paradigma diferente, de fácil aplicação e que pudesse ser utilizado em diversos ambientes de trabalho e para ocupações variadas. A construção de um instrumento geral de estresse ocupacional foi sugerida em 1987, por Murphy e Hurrel, como sendo necessária. Elaborou-se então, a Escala de Estresse no Trabalho (EET), cujo processo de construção e validação encontra-se descrito a seguir.

\section{Método}

Na presente seção, as diversas etapas seguidas na construção da escala serão apresentadas, bem como os procedimentos para a validação da mesma.

\section{Construção da escala}

\section{Construção dos itens}

Os itens da Escala de Estresse no Trabalho (EET) foram elaborados a partir da análise da literatura sobre estressores organizacionais de natureza psicossocial e sobre reações psicológicas ao estresse ocupacional bem como da análise de instrumentos já existentes. Neste último caso, 40\% dos itens basearam-se nos estressores abordados na versão brasileira do OSI (Swan, Moraes, \& Cooper, 1993) e no instrumento de Rizzo, House e Lirtzman (1970) sobre conflito e ambigüidade de papéis. 
Cada item da EET aborda tanto um estressor quanto uma reação ao mesmo. A decisão de conjugar estressor e reação deve-se à convicção do papel central da percepção como mediadora do impacto do ambiente de trabalho. Como foi discutido anteriormente, um fator organizacional constitui-se num estressor quando ele é percebido como tal pelo sujeito (Jex, 1998; Lazarus, 1995).

Dada a preocupação de construir um instrumento econômico e geral, os itens foram elaborados de forma a constituir um fator geral, contendo estressores variados e reações freqüentemente associadas aos mesmos. Com base em cada estressor organizacional de natureza psicossocial, citado freqüentemente na literatura (sobrecarga de trabalho, conflito entre papéis, ambigüidade de papéis, relacionamento interpessoal no trabalho, fatores de desenvolvimento na carreira e autonomia/controle no trabalho), foram elaborados itens, os quais contemplavam também, uma reação ao estressor. Os itens foram desenvolvidos de forma que pudessem ser aplicados a ambientes organizacionais diversos e a ocupações variadas, como "sinto-me incomodado por ter que realizar tarefas que estão além de minha capacidade” e “o tempo insuficiente para realizar meu volume de trabalho deixa-me nervoso".

\section{Análise de juízes}

Obteve-se assim, um total de 29 itens, os quais foram apresentados a um psicólogo organizacional e a um administrador, para que fosse avaliada a pertinência de cada um ao construto de estresse ocupacional e também a abrangência para aplicação em diversos contextos organizacionais. Como critério para manter o item na escala, considerou-se a concordância de $100 \%$ dos juízes, ou seja, os dois avaliadores precisariam concordar em suas respostas. Os 29 itens foram mantidos, pois houve concordância total entre os dois juízes quanto à pertinência e à abrangência. Outros dois itens foram inseridos por sugestão de um dos avaliadores.

Os 31 itens foram dispostos de forma aleatória em folheto com instruções apropriadas para auto-administração. Foi utilizada uma escala de concordância de cinco pontos: 1 (discordo totalmente), 2 (discordo), 3 (concordo em parte), 4 (concordo) e 5 (concordo totalmente).

\section{Análise semântica}

A fim de verificar se os itens propostos e as instruções apresentadas eram compreensíveis, o instrumento foi aplicado em dois grupos de três pessoas, todos funcionários de organizações públicas ou privadas, sendo que duas tinham segundo grau completo, três tinham superior incompleto e uma delas, superior completo. Foi solicitado a cada participante que respondesse ao questionário. Ao terminarem, foi feita uma discussão de grupo, na qual foi solicitado aos sujeitos que apontassem suas dificuldades em relação às instruções e aos termos presentes nos itens. A análise dos dados colhidos levou à modificação de termos em três itens. As instruções foram compreendidas pelos sujeitos tal como foram apresentadas e foram mantidas.

\section{Validação}

\section{Amostra}

A amostra utilizada para a validação do instrumento foi composta por 437 trabalhadores (249 homens e 188 mulheres) de diferentes organizações, públicas e privadas, localizadas no Distrito Federal e no estado de São Paulo. Os respondentes tinham idade média de 36 anos (desvio-padrão $=10,1)$ e nível de escolaridade variando do segundo grau incompleto (2,5\%) ao superior completo (60,5\%). Quanto à função desempenhada, 20,8\% eram analistas, 16,6\%, auxiliares/assistentes e 12,8\%, gerentes. O tempo médio de trabalho na organização foi de 11,4 anos (desvio-padrão = 8,7).

\section{Procedimento}

O instrumento foi administrado individualmente nas próprias organizações, em locais apropriados para a atividade, e coletivamente, em duas turmas de alunos de MBI formadas por empregados de empresas públicas e privadas.

\section{Análise dos dados}

A fim de verificar a dimensionalidade do instrumento, foi realizada análise exploratória dos dados e análise de componentes principais. Posteriormente, utilizou-se a técnica dos eixos principais com rotação oblíqua. O pressuposto inicial foi de que a escala era formada por um único fator. A fim de assegurar que cada item representasse o construto subjacente ao fator, foi estipulada uma carga fatorial mínima de 0,45 para aceitar o item. O cálculo da precisão da escala foi realizado por meio do coeficiente alfa de Cronbach.

\section{Resultados}

A partir da análise de componentes principais obtevese o índice Kaiser-Meyer-Olkin (KMO =0,91) e o Scree Plot. Este último revelou a existência de um único fator, o qual explicou $28 \%$ da variância. A análise fatorial com rotação oblíqua mostrou que o fator encontrado está composto por 23 itens.

A Tabela 1 aponta as cargas fatoriais para cada item do fator. No total, oito itens foram eliminados por apresentarem carga fatorial inferior a 0,45 , valor estipulado para aceitação do item na escala. A escala ficou composta por 23 itens e obteve um coeficiente alfa de 0,91.

\section{Discussão}

O objetivo deste trabalho consistiu em construir e validar um instrumento de estresse ocupacional geral, de fácil aplicação e que pudesse ser utilizado em diversos ambientes de trabalho e para ocupações variadas. Assim, partiu-se do pressuposto de que a escala seria formada por um único fator composto por estressores variados e reações emocionais diversas. A análise fatorial confirmou a idéia inicial de que os itens pudessem ser agrupados em um único fator. Mesmo assim, tentou-se uma solução diferente com 2 fato- 
Tabela 1

Cargas fatoriais para solução com um fator

\begin{tabular}{|c|c|}
\hline Item & Carga \\
\hline 20. Fico irritado por ser pouco valorizado por meus superiores & 0,684 \\
\hline 26. Tenho estado nervoso por meu superior me dar ordens contraditórias & 0,676 \\
\hline $\begin{array}{l}\text { 24. A falta de compreensão sobre quais são minhas responsabilidades neste trabalho tem causado } \\
\text { irritação }\end{array}$ & 0,666 \\
\hline 16. Sinto-me incomodado com a comunicação existente entre mim e meu superior & 0,655 \\
\hline 27. Sinto-me irritado por meu superior encobrir meu trabalho bem feito diante de outras pessoas & 0,640 \\
\hline 8. Tenho me sentido incomodado com a falta de confianç a de meu superior sobre o meu trabalho & 0,620 \\
\hline 17. Fico irritado com discriminação/favoritismo no meu ambiente de trabalho & 0,619 \\
\hline 5. O tipo de controle existente em meu trabalho me irrita & 0,590 \\
\hline 10. Sinto-me incomodado com a falta de informações sobre minhas tarefas no trabalho & 0,585 \\
\hline 30. Fico incomodado por meu superior evitar me incumbir de responsabilidades importantes & 0,576 \\
\hline 7. A falta de autonomia na execução do meu trabalho tem sido desgastante & 0,570 \\
\hline 4. A forma como as tarefas são distribuí das em minha área tem me deixado nervoso & 0,553 \\
\hline 19. Fico de mau humor por me sentir isolado na organização & 0,549 \\
\hline 23. A competição no meu ambiente de trabalho tem me deixado de mau humor & 0,547 \\
\hline 14. Fico de mau humor por ter que trabalhar durante muitas hor as seguidas & 0,542 \\
\hline 21. As poucas perspectivas de crescimento na carreira tem me deixado angustiado & 0,537 \\
\hline 12. Sinto-me incomodado por meu superior tratar-me mal na frente de colegas de trabalho & 0,529 \\
\hline 9. Sinto-me irritado com a deficiência na divulgação de informações sobre decisões organizacionais & 0,527 \\
\hline 22. Tenho me sentido incomodado por trabalhar em tarefas abaixo do meu nível de habilidade & 0,522 \\
\hline 28. O tempo insuficiente para realizar meu volume de trabalho deixa -me nervoso & 0,486 \\
\hline 11. A falta de comunicação entre mim e meus colegas de trabalho deixa -me irritado & 0,484 \\
\hline 18. Tenho me sentido incomodado com a deficiência nos treinamentos para capacitação profissional & 0,477 \\
\hline 13. Sinto-me incomodado por ter que realizar tarefas que estão além de minha capacidade & 0,467 \\
\hline 2. A falta de capacitação para a execução das minhas tarefas tem me deixado nervoso & 0,394 \\
\hline 3. A quantidade de trabalho tem me deixado cansado & 0,382 \\
\hline 15. Sinto nervosismo em ter que me manter atualizado com as mudanças tecnol ógicas & 0,367 \\
\hline 31. As estratégias utilizadas para introduzir novas tecnologias me deixam angustiado & 0,366 \\
\hline 25. Sinto-me de mau humor com "fofocas" no meu ambiente de trabalho & 0,362 \\
\hline $\begin{array}{l}\text { 29. Fico de mau humor com brincadeiras de mal gosto que meus colegas de trabalho fazem uns com os } \\
\text { outros }\end{array}$ & 0,317 \\
\hline 1. Os prazos estabelecidos para a realização das minhas tarefas são satisfatórios & 0,288 \\
\hline 6. Em meu trabalho não há pressão & 0,264 \\
\hline
\end{tabular}

res. Os resultados, porém, não foram satisfatórios, obtendose fatores com conteúdos difíceis de interpretar, o que confirmou a idéia inicial de uma escala unifatorial.

A exclusão de oito itens da versão final da escala não prejudicou o conteúdo da mesma, pois cada um dos eliminados tinha algum correspondente entre os itens mantidos (por exemplo, o item eliminado "a falta de capacitação para a execução das minhas tarefas tem me deixado nervoso” tinha como correspondente "sinto-me incomodado por ter que realizar tarefas que estão além de minha capacidade”, o qual permaneceu na escala).

A EET possui características psicométricas satisfatórias e pode contribuir tanto para pesquisas sobre o tema quanto para o diagnóstico do ambiente organizacional, completando informações advindas de outros instrumentos. Considerando sua utilidade prática, propôs-se uma versão reduzida, mais rápida e econômica, composta por 13 itens, representantes dos principais estressores organizacionais e reações psicológicas gerais. O coeficiente alfa desta versão é de 0,85 . Os itens que compõem a versão reduzida são: 4, 7, 8, 9, 13, 18, 19, 20, 21, 22, 23, 24 e 28.

O objetivo de validação de uma escala de estresse ocupacional foi alcançado e duas versões do instrumento propostas, ambas com padrões psicométricos sólidos. Numa área de estudo em expansão, mas com diversas limitações em relação aos instrumentos de medida, a EET consiste numa alternativa tanto para investigações empíricas quanto para trabalhos aplicados, podendo ser usada para organizações e cargos variados. Comumente, os instrumentos utilizados para avaliar o estresse ocupacional consideram ou uma escala de estressores ou uma escala de reações e, quando consideram as duas, não estabelecem um nexo entre elas. A EET evita 
fazer duas avaliações separadas e considera a percepção do indivíduo, o que vai ao encontro das críticas referentes a abordagens que enfocam estressores ou reações isoladamente e, desta forma, preenche algumas lacunas existentes nos instrumentos de avaliação de estresse ocupacional.

A literatura tem apontado freqüentemente os efeitos negativos do estresse, tanto para a saúde e o bem-estar individual quanto para a efetividade organizacional. Esta escala pode ser utilizada como ferramenta no diagnóstico do ambiente de trabalho das organizações, orientando medidas que visem à qualidade de vida dos funcionários.

Apesar das contribuições que a EET pode trazer, algumas limitações deste instrumento podem ser apontadas. Obviamente, por se tratar de uma escala geral, cujos itens contêm estressores variados e reações emocionais freqüentemente associadas aos mesmos, a EET pode ser pouco eficaz quando se quer enfatizar estressores isolados ou quando se deseja investigar a influência de determinadas variáveis situacionais e individuais sobre o estresse ocupacional. Kahn e Byosiere (1992), em sua revisão sobre a relação entre comportamento Tipo A e estresse ocupacional, salientam a existência de divergências entre estudos que abordam esses dois construtos e apontam, como uma das justificativas para essa inconsistência, a utilização de medidas diferentes e limitadas de estressores organizacionais. Para os autores, a influência do comportamento tipo A sobre o estresse ocupacional ocorre com alguns tipos de estressores apenas. Há possibilidade de que, conforme sugerido por Kahn e Byosiere (1992) em relação ao comportamento Tipo A, outras variáveis possivelmente preditoras do estresse ocupacional também se correlacionem com estressores organizacionais específicos.

Quanto à variância explicada pelo fator que compõe a escala, o valor encontrado foi modesto. Conforme discutido anteriormente, este instrumento consiste numa medida bastante geral do estresse ocupacional. A desvantagem dessa generalidade é que se podem perder aspectos mais específicos de determinadas ocupações, organizações, reações individuais e outras variáveis que constituem o fenômeno em questão. Por outro lado, medidas que tentam contemplar toda essa complexidade, como o OSI (Cooper, Sloan, \& Williams, 1988), têm apresentado problemas de construção e validação.

Pesquisas futuras poderiam desenvolver escalas de estresse ocupacional que se baseassem na abordagem que guiou a construção da EET, mas que considerassem separadamente as diferentes categorias de estressores, o que resultaria em escalas fatoriais ou unifatoriais, dependendo do número de estressores específicos estudados. As emoções associadas aos estressores organizacionais também poderiam ser melhor exploradas, investigando-se se determinadas emoções são mais características de determinados estressores. Outras pesquisas poderiam considerar a técnica da validação convergente, a qual parte do princípio de que para demonstrar a validade de construto de um instrumento é preciso determinar se este se correlaciona significativamente com ou- tras variáveis com as quais o construto medido deveria, pela teoria, estar relacionado (Pasquali, 1999). Desta forma, a EET poderia ser aplicada juntamente com outras medidas que se propõem a avaliar o estresse ocupacional e verificar a correlação entre os resultados obtidos. Seria interessante também, aplicar a EET e, posteriormente, entrevistar uma parte de sujeitos da amostra para explorar se os níveis de estresse avaliados pela escala correspondem aos níveis descritos pelos indivíduos na entrevista.

\section{Referências}

Beehr, T. A. (1998). Research on occupational stress: an unfinished enterprise. Personnel Psychology, 51, 835-844.

Caplan, R. D., \& Jones, K. W. (1975). Effects of workload, role ambiguity, and Type A personality on anxiety, depression, and heart rate. Journal of Applied Psychology, 60, 713-719.

Cavanaugh, M. A., Boswell, W. R., Roehling, M. V., \& Boudreau, J. W. (2000). An empirical examination of self-reported work stress among U.S. managers. Journal of Applied Psychology, 85, 65-74.

Cohen, S., Kessler, R. C., \& Gordon, L. U. (1995). Measuring stress. Oxford: Oxford University Press.

Cooper, C. L., \& Cartwright, S. (2001). Organizational management of stress and destructive emotions at work. In R. L. Payne \& C. L. Cooper (Orgs.), Emotions at work: theory, research and applications for management (pp. 269-280). Chichester: John Wiley \& Sons.

Cooper, C. L., Dewe, Ph. J., \& O’Driscoll, M. P. (2001). Organizational stress. A review and critique of theory, research and applications. Londres: Sage.

Cooper, C., Sloan, S., \& Williams, S. (1988). Occupational stress indicator management guide. Londres: Thorbay.

Evans, O., \& Steptoe, A. (2001). Social support at work, heart rate, and cortisol: A self-monitoring study. Journal of Occupational Health Psychology, 6, 361-370.

Evers, A., Frese, M., \& Cooper, C. L. (2000). Revisions and further developments of the occupational stress indicator: LISREL results from four Dutch studies. Journal of Occupational and Organizational Psychology, 73, 221-240.

Fried, Y., Ben-David, H. A., Tiegs, R. B., Avital, N., \& Yeverechyahu, U. (1998). The interactive effect of role conflict and role ambiguity on job performance. Journal of Occupational and Organizational Psychology, 71, 19-27.

Guimarães, L. A. M., \& McFaddeb, M. A. J. (1999). Validação para o Brasil do SWS Survey - Questionário sobre estresse, saúde mental e trabalho. In L. A. M. Guimarães \& S. Grubits (Orgs.), Série saúde mental e trabalho (vol. 1, pp. 153-168). São Paulo: Casa do Psicólogo.

Glowinkowski, S. P., \& Cooper, C. L. (1987). Managers and professionals in business/industrial settings: the research evidence. In J. M. Ivancevich \& D. C. Gangster (Orgs.), Job stress: from theory to suggestion. Nova York: Haworth.

Iwata, N., \& Suzuki, K. (1997). Role stress - mental health relations in Japanese bank workers: a moderating effect of social support. Applied Psychology: An International Review, 45, 207-218.

Jamal, M. (1990). Relationship of job stress and type A behavior to employee's job satisfaction, organizational commitment, psychosomatic problems and turnover motivation. Human Relations, 8, 727-738.

Jex, S. M. (1998). Stress and job performance. Londres: Sage.

Jex, S. M., \& Elacqua, T. C. (1999). Self-esteem as a moderator: A comparison of global and organization-based measures. Journal of Occupational and Organizational Psychology, 72, 71-81. 
Jones, F., \& Kinman, G. (2001). Approaches to studying stress. In F. Jones \& J. Bright (Orgs.), Stress: myth, theory and research (pp. 17-45). Londres: Prentice Hall.

Kahn, R. L., \& Byosiere, P. (1992). Stress in organizations. In M. D. Dunnete \& L. M. Hough (Orgs.), Handbook of industrial and organizational psychology (v. 3, pp. 571-650). Palo Alto: Consulting Psychologists.

Krause, N., \& Stryker, S. (1984). Stress and well-being: the buffering role of locus of control beliefs. Social Science and Medicine, 18, 783-790.

Lazarus, R. S. (1995). Psychological stress in the workplace. In R. Crandall, \& P. L. Perrewé (Orgs.), Occupational stress: A Handbook (pp. 3-14). Washington: Taylor \& Francis.

Lazarus, R. S., \& Folkman, S. (1984). Stress. Appraisal, and coping. Nova York: Springer.

Lim, C. S., Ong, C. N., \& Phoon, W. O. (1987). Work stress of firemen as measured by heart rate and catecholamine. Journal of Human Ergology, 16, 209-218.

Lyne, K. D., Barret, P. T., Williams, C., \& Coaley, K. (2000). A psychometric evaluation of the occupational stress indicator. Journal of Occupational and Organizational Psychology, 73, 195-220.

Mackie, K. S., Holahan, C. K., \& Gottlieb, N. H. (2001). Employee involvement management practices, work stress, and depression in employees of a human service residential care facility. Human Relations, 54, 1065-1092.

Marino, K. E., \& White, S. E. (1985). Departmental structure, locus of control, and job stress: the effect of moderators. Journal of Applied Psychology, 70, 782-784.

Murphy, L. R., \& Hurrell, J. J. (1987). Stress measurement and management in organizations: Development and current status. In A. W. Riley \& S. J.
Zaccaro (Orgs.), Occupational stress and organizational effectiveness (pp. 25-51). Nova York: Praeger.

Pasquali, L. (1999). Testes referentes a construto: teoria e modelo de construção. In L. Pasquali (Org.), Instrumentos psicológicos: manual prático de elaboração (pp. 37-71). Brasília: LabPAM/ IBAPP.

Pollard, T. M. (2001). Changes in mental well-being, blood pressure and total cholesterol levels during workplace reorganization: The impact of uncertainty. Work and stress, 15, 14-28.

Quick, J. C., Quick, J. D., Nelson, D. L., \& Hurrell, J. J. (1997). Preventive stress management in organizations. Washington: American Psychological Association.

Rizzo, J. R., House, R. J., \& Lirtzman, S. I. (1970). Role conflict and ambiguity in complex organizations. Administrative Science Quarterly, 15, 150-163.

Stanley, R., \& Burrows, G. (2001). Varieties and functions of human emotion. In R. L. Payne \& C. L. Cooper (Orgs.), Emotions at work: Theory, research and applications for management (pp. 3-19). Chichester: John Wiley and Sons.

Swan, J. A., Moraes, L. F. R., \& Cooper, C. L. (1993). Developing the occupational stress indicator (OSI) for use in Brazil: A report on the reliability and validity of the translated OSI. Stress Medicine, 9, 247-453.

Tamayo, A., Paschoal, T., Rego, V. B., \& Ferreira, I. F. R. (2002). Relação entre estresse ocupacional e autoconceito profissional [Resumo]. In Sociedade Brasileira de Psicologia (Org.), Resumos de comunicações científicas, XXXII Reunião Anual de Psicologia (p. 309), Florianópolis: Autor.

Wang, J. L., \& Patten, S. B. (2001). Perceived work stress and major depression in the Canadian employed population, 20-49 years old. Journal of Occupational Health Psychology, 6, 283-289.

Tatiane Paschoal é mestre em Psicologia Social e do Trabalho. E-mail: tatipas@yahoo.com

Álvaro Tamayo, doutor em Psicologia pela Université de Louvain, é professor no Departamento de Psicologia Social e do Trabalho, Universidade de Brasília. Endereço para correspondência: Departamento de Psicologia Social e do Trabalho, Universidade de Brasília, Asa Norte; Brasília, DF; CEP 70910-900. E-mail: tamayo@unb.br 